\title{
On inferring hybridity from morphological intermediacy
}

\author{
Paul Wilson ${ }^{1}$
}

\begin{abstract}
Summary
Wilson, P.: On inferring hybridity from morphological intermediacy. - Taxon 41: 11-23. 1992. - ISSN 0040-0262.

The type of intermediacy that separates hybridity from divergence is not multivariate intermediacy; it is the coincidence of intermediate character states. For the purpose of showing hybridity, analyses should distinguish between the two types of intermediacy. (1) Hybrid indices fail to do so. (2) Principal components analysis does so only in an ambiguous way. (3) Pictorialized scatter diagrams properly present the evidence for an interpretation that is intuitive. (4) Counting characters as intermediate or not-intermediate is an explicit approach that allows for statistical evaluation given that certain assumptions are made. Simulated data representing hybridity and divergence are presented to illustrate each method and to provide a prototype of how to document hybridity using the favored methods.
\end{abstract}

Hybridization results in intermediacy between parents in many characters. Several methods are in common usage for presenting intermediacy to demonstrate hybridity (literature to be reviewed in Discussion). 'Hybrid indices' and 'principal components analysis' have been repeatedly used in documenting hybridity. Although other researchers have criticized these methods for being statistically biased, I believe them to be fundamentally inappropriate in that they do not show the type of intermediacy that is unique to the results of hybridization. Two other approaches, 'pictorialized scatter diagrams' and what I call the 'character count procedure', are appropriate. I will refine these two methods.

To show that a plant is of hybrid origin means to distinguish that possibility from the alternative, which is origin through divergence (Wagner, 1969). Consider Fig. 1 and 2, which show contrasting historical scenarios resulting in contrasting character patterns. Fig. 1 illustrates a kind of divergence where ancestral species did not go extinct - I call the result a 'phyletic series'. In Fig. 1a, a new species diverged acquiring pigment in its knobs; in Fig. 1b, a second new species diverged with an enlargement of its central spot. Fig. 2 illustrates hybridization - I call the result a 'reticulate series' In Fig. $2 \mathrm{a}$, there are two species that have diverged in spine length and intensity of shading; in Fig. 2b, they hybridize, and the new form is intermediate in both characters. The central species of a phyletic series (B) resembles the end species (A and C) in separate characters. The central species of a reticulate series (F) splits the difference between the conditions of the end species ( $D$ and $E$ ) in each character. These are two very different kinds of intermediacy: overall intermediacy, and characterby-character intermediacy. Methods that fail to discriminate between the two types of intermediacy are not useful in distinguishing hybridity from divergence, in contrast to methods that specifically demonstrate character-by-character intermediacy.

Although the figures illustrate only two characters each, an inference of hybridity should be based on the number of characters that are intermediate, and two characters would not be considered strong evidence. This is because it is possible for there to be

\footnotetext{
I Department of Ecology and Evolution, State University of New York, Stony Brook, NY 11794, U.S.A.
} 
deviations from the ideal difference between phyletic and reticulate series, for at least three reasons.

(1) For any one character, a phyletic series may look like a reticulate series because there have been evolutionary changes in adjacent segments of the phylogeny. For instance, a species with small leaves might give rise to a second species with large leaves, and that second species might give rise to a third species with very large leaves. To resolve this uncertainty, examine many separate characters. A large number of characters is important because it is not reasonable to invoke the same directional trend in different characters. The distinctness of the characters is important because unrecognized pleiotropic or functional character correlations will mislead one into assuming more genetic intermediacy than actually exists.

(2) A reticulate series may deviate from the expected pattern when there is extreme dominance or epistasis. The expectation of intermediacy is based on an assumption that most alleles combine with a predominantly additive effect. To the extent that there is dominance or epistasis, it tends to hide reticulate series. Unless one knows the genetic basis of character expression (as for allozymes), nothing can be done about this problem since the same pattern results from divergence as from hybridity (see Rieseberg \& al., 1988 for an analysis of molecular data). One simply must make an interpretation based on as many characters as possible. Inevitably, some characters will not be intermediate in any hybrid.

(3) Breeding past the $F_{1}$ generation, especially backcrossing, may alter a pattern of reticulate intermediacy. Recombination of alleles will to some extent (depending on the genetics), lead to the expression of parental rather than medial character states. Unless there is polyploidization, subsequent sexual reproduction tends to obscure reticulate series. The morphological approach discussed here works best for situations in which most of the hybrids are $F_{1}$ 's or polyploid derivatives (McDade, 1990; contra

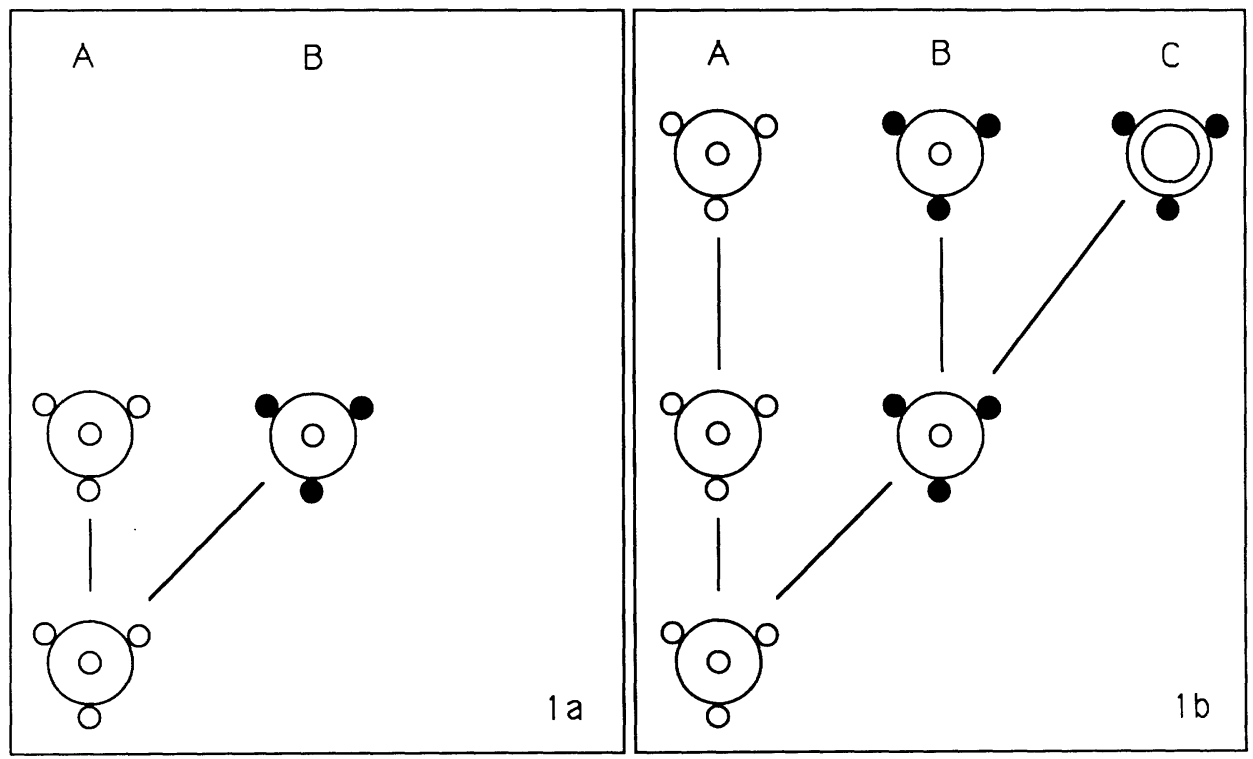

Fig. 1. Idealized scenario envisioned for the origin of a phyletic series. In la, species A and B diverged in knob pigmentation. In $1 \mathrm{~b}$, species $\mathrm{C}$ diverged from species $\mathrm{B}$ in central spot size. species B shares some characters with species A and others with species $C$. 
Funk, 1985); more complex origins might be better resolved by knowledge of molecular genetic markers throughout the ranges of the species involved.

Despite these problems, systematists have a long record of successfully identifying hybrids as such. Morphological intermediacy has been a primary criterion. Important evidence has also come from cytology, sterility determinations, enzyme polymorphisms, teratology, micro-biogeography, and comparison against artificial hybrids (Wagner, 1983). In documenting a case for hybridity, all of these forms of evidence can be valuable. In this paper, I will critique four methods of showing morphological intermediacy in terms of their ability to distinguish phyletic from reticulate series.

\section{Data}

I wrote two PASCAL programs. Each produces a data set. One program simulates divergence, and the other simulates hybridization. These simulations allow me to illustrate the methods to be discussed using data that can be thought of as coming from a known phyletic series and a known reticulate series.

The divergence program does the following. (1) Species 1 is established, de novo, having 20 characters, all with a value of 10 units. (2) Species 2 is created from species 1 by divergence in a random selection of 15 of the 20 characters. Each of the 15 is modified either positively or negatively at random. Each is modified a random amount on a uniform scale for up to 5 units. Species 1 itself goes unchanged. (3) Species 3 is subsequently created by divergence in a similar manner, again from species 1 (though divergence from species 2 would still create a phyletic series). The 15 characters in which species 3 diverges are separately chosen at random, so by chance a few are the same as were modified in the divergence of species 2. (4) Samples of 50 individuals are drawn from all three species: for each character, the character state of an individual is made to deviate from its species's value at random on a normal distribution with a standard deviation of 1 unit. (5) Across species, randomly generated multipliers between 0.1 and 10 are used to transform the variates out of the

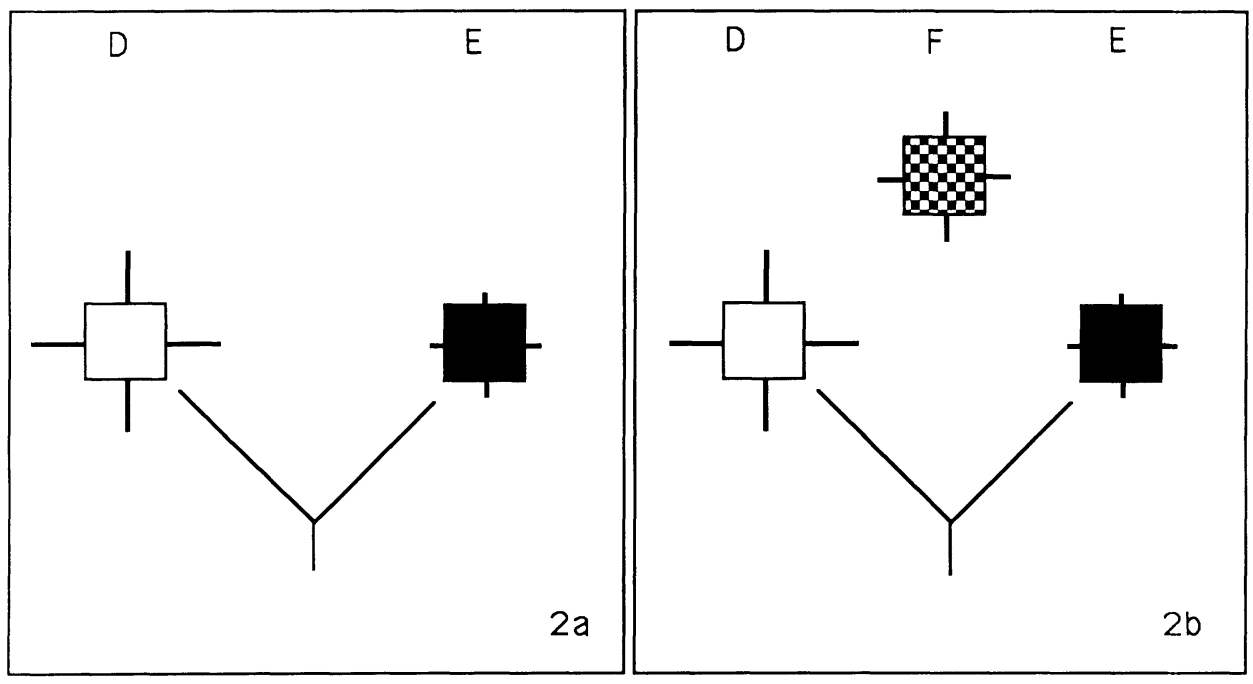

Fig. 2. Idealized scenario envisioned for the origin of a reticulate series. In $2 \mathrm{a}$, species $\mathrm{D}$ and $\mathrm{E}$ have diverged in spine length and pigmentation. In $2 b$, they hybridize to form species F. Species $\mathrm{F}$ is between $\mathrm{D}$ and $\mathrm{E}$ in each character. 
original common units into unit scales unique to each character - this step makes the data seem more realistic but does not change any of the results.

The hybridization program does the following. (1) Species 4 and species 5 are established, differing in 15 characters. The amount of the difference is random on a uniform distribution for up to 10 units; the values range from 5 to 15. (2) For each character, a dominance factor is randomly generated from a normal distribution with a mean of 0.5 (no dominance) and a standard deviation of 0.25 (a factor deviating from 0.5 by 2 standard deviations would result in complete dominance). Results from a second, higher level of dominance, standard deviation $=0.50$, will also be mentioned below. (3) Species 6 is created by hybridization: one individual from species 4 and one from species 5 are drawn at a time; for each character an individual's character state is made to deviate from its species's value at random on a normal distribution with a standard deviation of 1 unit; the two individuals are crossed - for each character, the parental character states are added together after being weighted by the dominance factor associated with that character. This is done 50 times to form a sample of 50 individuals. (4) Samples of 50 deviant individuals are separately drawn from species 4 and from species 5. (5) Across species, the variates of each character are transformed into unique units as before.

\section{Hybrid index analysis}

A hybrid index is a quantity calculated for an individual that places it on a scale made up of the combined ranges of all the characters (Anderson, 1949). For equal weighting of characters, variates were rescored such that the new range for each character was from 0 to 1 and the direction of increase for all characters was uniform: from each variate, I subtracted the minimum value for that character; I then divided by the range; and, depending on the original direction of increase, I did or did not additively invert the variates of a character around 0.5 . Hybrid index values were calculated for each individual as the mean of transformed variates across all characters. These values were plotted by frequency.
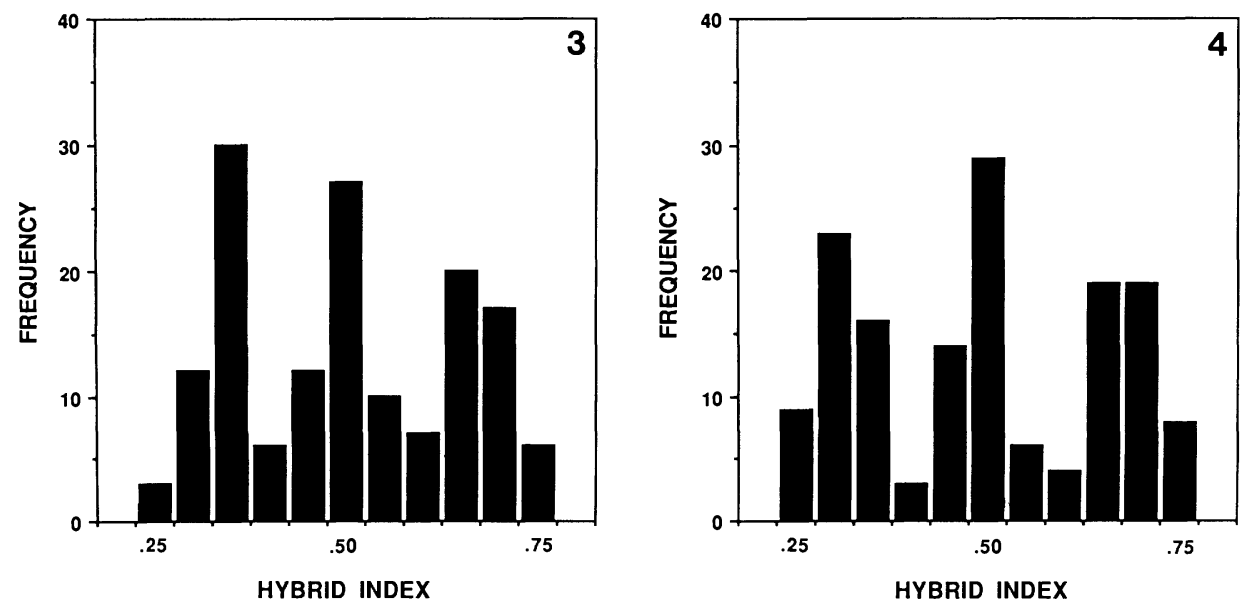

Fig. 3. Histogram of hybrid indices for data from the simulated phyletic series; 150 individuals pooled.

Fig. 4. Histogram of hybrid indices for data from the simulated reticulate series; 150 individuals pooled. 
The hybrid index histograms reveal an intermediate peak for both the phyletic series (Fig. 3) and the reticulate series (Fig. 4). What cannot be told from the histograms is that the intermediate peaks result from different types of patterns. The intermediate peak in the phyletic series represents overall intermediacy - similarities between the central species and one end species counteract similarities between the central species and the other end species. The intermediate peak in the reticulate series represents character-by-character intermediacy - many of the characters of the central species have values between those of the end species. Hybrid indices do not distinguish hybridity from divergence.

If hybridity is already known, hybrid indices might be useful in flagging individuals that are not as equally intermediate as others, plants that might be worth studying further (Davis \& Heywood, 1963). Hybrid indices are potentially useful in showing backcrossing, and, when a population is sampled at random, hybrid index histograms can be used to show the structure of a hybrid swarm. Hatheway (1962) and Goodman (1967) discuss character weighting for the hybrid index. Gay (1960) provides an elaboration on hybrid index analysis for comparing populations differing in the amount of hybridization.

\section{Principal components analysis}

Principal components analysis is a method whereby the variance in a number of characters is resolved onto synthetic axes by taking into account the covariance between characters. The first principle components axis is made to explain all the variance that can be explained in one dimension, the second axis is made to explain the maximum amount remaining in a second independent dimension, and so on (Pimentel, 1979). The PRINCOMP STD procedure of SAS (Anonymous, 1985) was used to determine the placement of individuals on the first two principal component axes based on standardized characters.

Position on the axes are plotted for the phyletic series in Fig. 5, for the reticulate series with moderate dominance in Fig. 6, and for the reticulate series with high dominance in Fig. 7. In all three figures, there is a group that is intermediate between two other groups on the first axis. In the phyletic series and in the reticulate series with high dominance there is a separation of groups on the second as well as the first axis. The results for the two axes can be explained in sequence.

(1) In the phyletic series, characters covary because character changes accumulate in a lineage as evolution proceeds. Not only do they accumulate within a segment of a phylogeny - in Fig. 1, the diagonal line between species A and B - they also accumulate through adjacent segments - through the two diagonals between species $\mathrm{A}$ and $\mathrm{C}$. Thus, character differences between species $\mathrm{A}$ and $\mathrm{B}$ can be incorporated into the same axes as differences between species B and C, and for similar reasons one group is between two other groups in Fig. 5. In a reticulate series, characters covary and hybrids are intermediate. Here again the three groups are separated on the first principal components axes, as shown in Fig. 6 and 7. Thus, intermediacy on the first axis does not distinguish between hybridity and divergence.

(2) In a reticulate series in which every character conforms to idealized expectations (alleles of additive effect and no recombination), all useful characters will be correlated - the first axis will explain all the variance among groups, though the second axis will still explain variance among individuals within groups (Fig. 6). In contrast, in a phyletic series, the second axis is important as well as the first axis (Fig. 5). This 
reflects the fact that different characters separate the central species from each of the two end species. One might, then, suppose that principal components analysis can be useful in inferring hybridity not through intermediacy itself but through the sufficiency of the first axis. However, even in a reticulate series, the second axis will often be important in separating groups; this is because any kind of deviation from idealized expectations will lead to covariation that is independent of the differences between parents and the intermediacy of hybrids. For instance, the reticulate series with high dominance closely resembles the phyletic series (compare Fig. 5 and 7). I do not recommend this technique, but, if principal components are used to infer hybridity, the evidence is the sufficiency of the first axis in separating groups, not mere intermediacy.

Principal components may be more useful in finding putatively hybridizing groups, locating the most likely parent of a known hybrid with several possible parents, or searching for possible backcrossed plants in a hybrid swarm (Wagner, 1983). Namkoong (1966) and Smouse (1972) provided refinements on this method and related techniques for analysis of hybrid swarms. Pimentel (1981) compared various ordination techniques in terms of how well they separated parents, hybrids, and
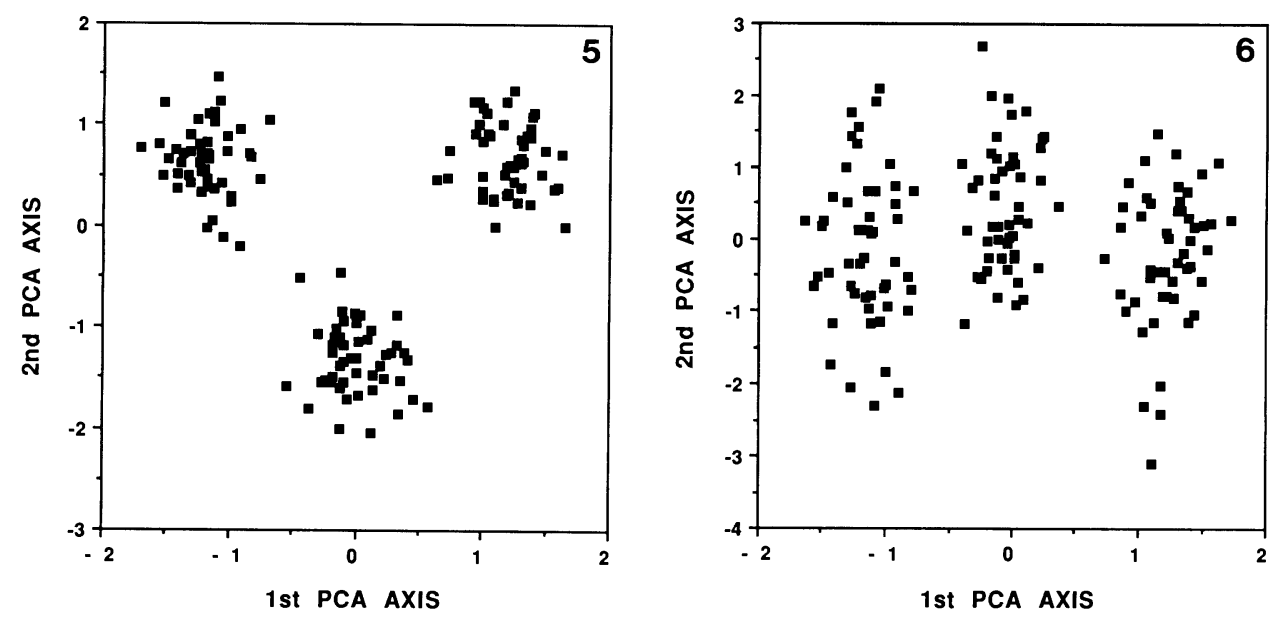

Fig. 5. Principal components analysis of data from the simulated phyletic series.

Fig. 6. Principal components analysis of data from the simulated reticulate series with low dominance.

Fig. 7. Principal components analysis of data from the simulated reticulate series with high dominance.

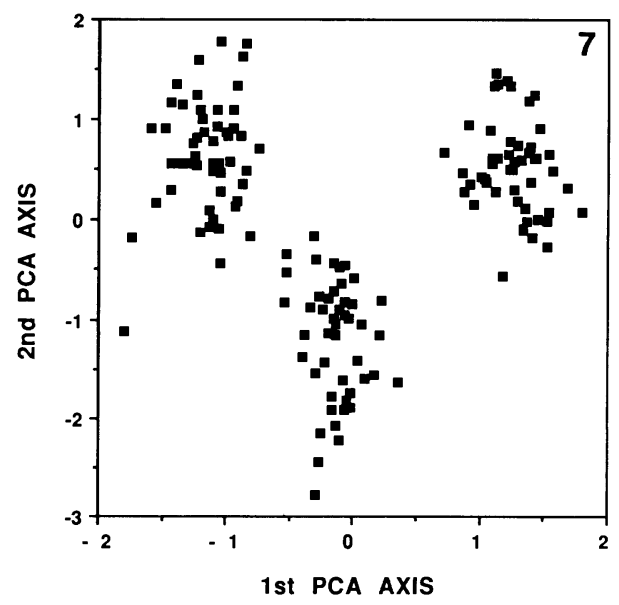


introgressants (also see Dancik \& Barnes, 1975; Adams, 1982). This family of techniques, along with hybrid indices, does not allow one to distinguish between hybridity and primary divergence.

\section{Pictorialized scatter diagrams}

A pictorialized scatter diagram is a graphical presentation of the character states of individuals, the data being recoded so that it is easy to see character-by-character intermediacy when it exists (Anderson, 1949). A well-drawn pictorialized scatter diagram facilitates interpretation. I recommend that the icons at one end of a series be unadorned (open squares without spines) and those at the other end be ornamented (filled squares with long spines); reticulate intermediates will then be balanced with a moderate level of ornamentation (half-filled squares with short spines), whereas

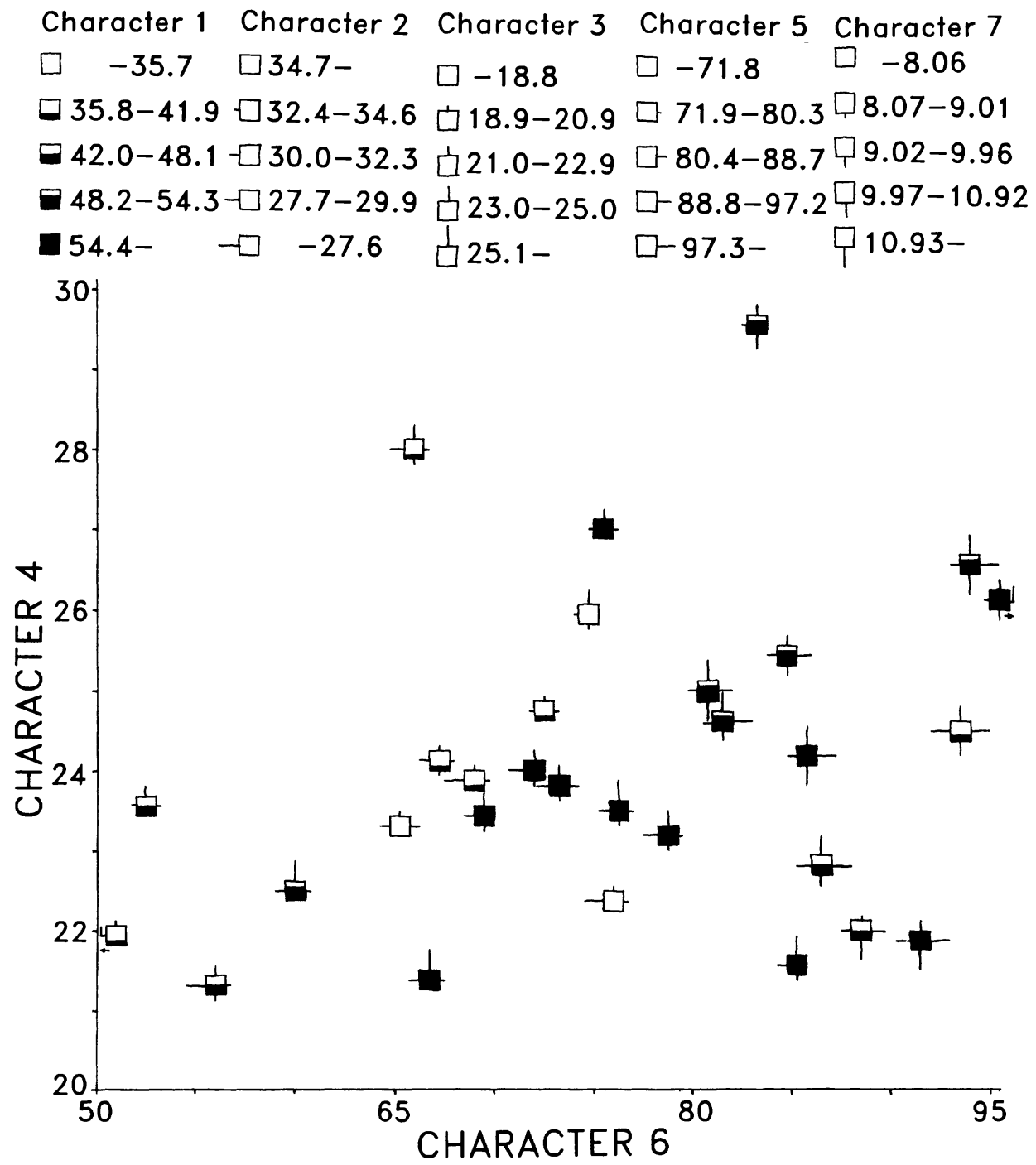

Fig. 8. Pictorialized scatter diagram of data from the simulated phyletic series. 
phyletic intermediates will be an odd mixture of accentuated and minimized features (some spines long, others absent). I used seven characters symbolized as follows: position along the horizontal axis, position along the vertical axis, blackening of the square, and length for each of four spines. The axis characters are treated as continuous. The others are coded into five classes: the range is divided into seven parts, then the first two and the last two are pooled, making the three central classes (where hybrids will fall) more prominent.

Fig. 8 shows a diagram for the phyletic series. Fig. 9 shows one for the reticulate series. The intermediates of Fig. 8 are unbalanced. The intermediates of Fig. 9 are orderly. There is no character-by-character intermediacy in Fig. 8. Hybridity is sup-
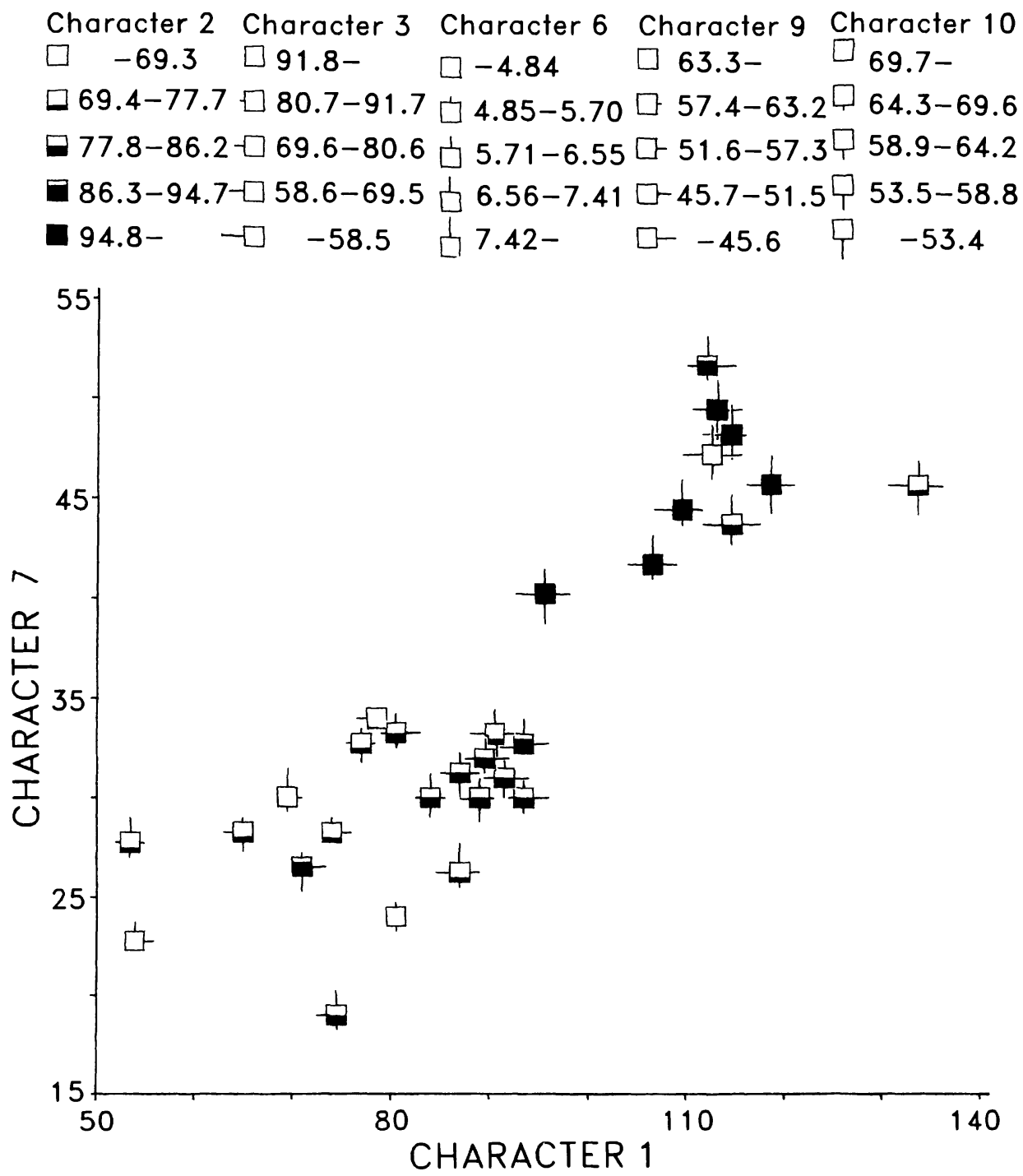

Fig. 9. Pictorialized scatter diagram of data from the simulated reticulate series. 
Table 1. Character count procedure (steps 3 and 4) for data from the simulated phyletic series. Means \pm standard deviations are presented; sample sizes were 50 throughout. "1 different from" indicates significantly differences between species 2 or 3 or both or neither based on Tukey multiple comparisons. "1 intermediate?" indicates whether or not species 1 was between 2 and 3 for that character; the tally of these indications is presented at the bottom of the column. Character 2 is not shown since it was not useful in separating species 2 and 3.

\begin{tabular}{ccccccc}
\hline $\begin{array}{c}\text { Character } \\
\text { No. }\end{array}$ & $\begin{array}{c}\text { Species } \\
2\end{array}$ & $\begin{array}{c}\text { Species } \\
1\end{array}$ & \multicolumn{2}{c}{$\begin{array}{c}\text { Species } \\
3\end{array}$} & $\begin{array}{c}1 \text { different } \\
\text { from }\end{array}$ & $\begin{array}{c}1 \text { inter- } \\
\text { mediate? }\end{array}$ \\
\hline 1 & $38.90 \pm 4.464$ & $58.11 \pm 4.847$ & $49.93 \pm 5.590$ & both & - \\
3 & $20.81 \pm 2.164$ & $23.11 \pm 2.496$ & $23.12 \pm 2.630$ & 2 & + \\
4 & $24.96 \pm 2.434$ & $24.57 \pm 2.067$ & $23.77 \pm 2.227$ & neither & + \\
5 & $73.15 \pm 7.378$ & $71.50 \pm 7.135$ & $103.75 \pm 5.836$ & 3 & - \\
6 & $61.32 \pm 8.366$ & $72.00 \pm 7.610$ & $93.13 \pm 7.023$ & both & + \\
7 & $8.21 \pm 0.845$ & $8.43 \pm 0.894$ & $11.09 \pm 0.908$ & 3 & + \\
8 & $37.56 \pm 3.105$ & $25.99 \pm 2.579$ & $25.77 \pm 2.427$ & 2 & + \\
9 & $94.60 \pm 9.403$ & $93.63 \pm 10.113$ & $112.11 \pm 10.319$ & 3 & - \\
10 & $42.87 \pm 4.148$ & $42.00 \pm 4.118$ & $21.86 \pm 3.936$ & 3 & + \\
11 & $1.86 \pm 0.216$ & $2.40 \pm 0.215$ & $2.33 \pm 0.278$ & 2 & - \\
12 & $62.83 \pm 5.461$ & $47.15 \pm 5.219$ & $48.27 \pm 4.306$ & 2 & - \\
13 & $21.83 \pm 2.452$ & $22.32 \pm 2.463$ & $23.08 \pm 2.342$ & neither & + \\
14 & $139.60 \pm 9.345$ & $93.48 \pm 10.211$ & $94.99 \pm 8.752$ & 2 & - \\
15 & $67.08 \pm 6.931$ & $67.44 \pm 5.821$ & $98.73 \pm 7.005$ & 3 & + \\
16 & $25.75 \pm 2.027$ & $23.25 \pm 2.595$ & $23.37 \pm 2.329$ & 2 & - \\
17 & $60.29 \pm 7.183$ & $74.49 \pm 6.131$ & $36.68 \pm 7.130$ & both & - \\
& & & & & & $8: 8$ \\
\hline
\end{tabular}

ported in Fig. 9 by a careful inspection of each feature of the central icons compared to the extreme icons.

The diagrams neither combine varying individuals into groups before considering deviations in the pattern of different characters (see next section) nor do they combine characters into indices before considering the deviations in the pattern of different individuals (as does the method of Wells, 1980) - the viewer is presented with both kinds of information. The chief limitations of the approach are that (1) the evaluation of that information remains subjective, and (2) the inclusion of many individuals or many characters make the diagrams excessively difficult to follow. Still, the diagrams remain useful in inferring hybridity (Davis \& Heywood, 1963). They can also be employed in showing the degree of intergradation in a hybrid swarm. Warwich \& al. (1989) plot pictorialized icons on principal component axes - this makes for a meaningful adjustment in the position of the icons, but, if the diagrams are done this way, I recommend more spines be used so that as many characters are shown individually as can be done with clarity.

\section{Character count procedure}

The character count procedure is an approach that has never to my knowledge been codified in the way I will present it. It is, nevertheless, implicitly part of most papers claiming to show hybridity. The analysis proceeds by steps rather than being con- 
Table 2. Character count procedure (steps 3 and 4) for data from the simulated reticulate series with limited dominance (explanation as in Table 1).

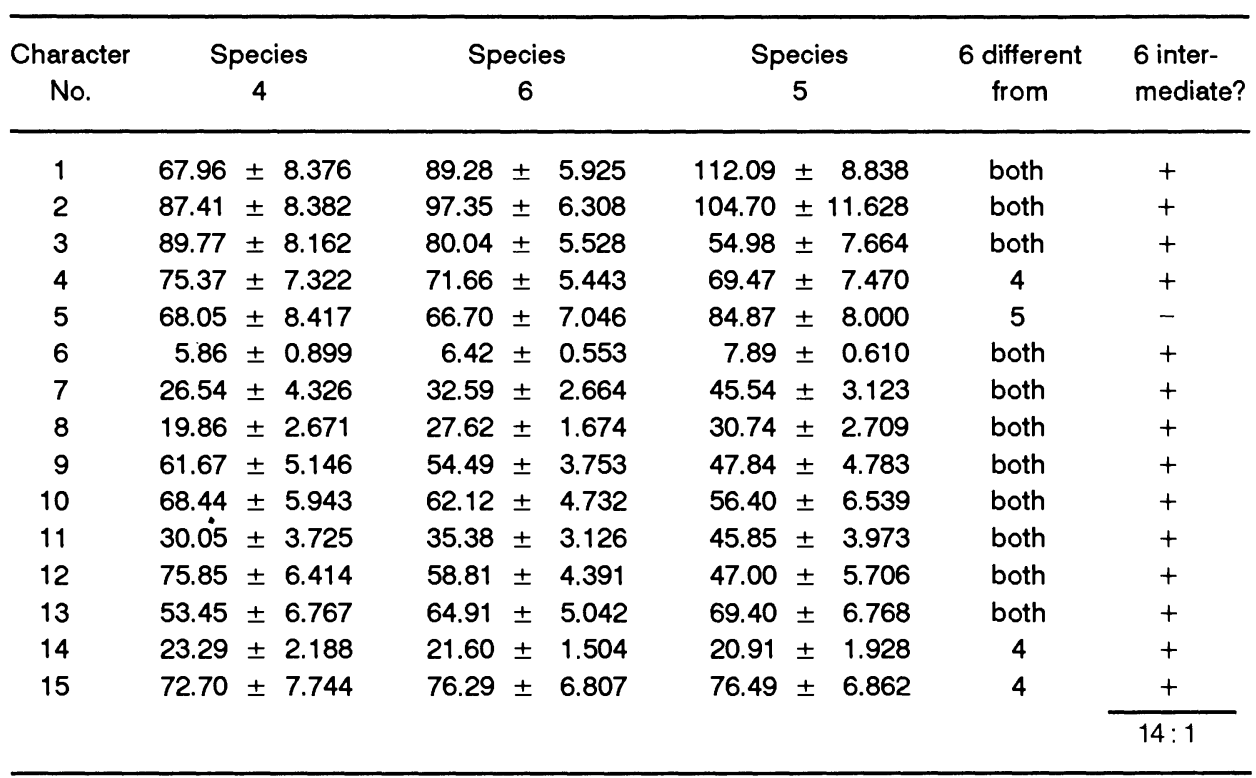

certed. (1) Place individuals in groups - two putative parental taxa and a putative hybrid taxon. Grouping can be done subjectively or with numerical techniques. It is often useful to have knowledge of the parental species in allopatry. (2) Select characters that separate the parents. Do this without considering how the characters combine in the putative hybrids. (3) For each character, determine and tabulate whether or not the value (e.g., mean size, color, etc.) for the hybrids is intermediate. Report on non-intermediate as well as intermediate characters (some authors have failed to do this, e.g., Sytsma \& Pippen, 1985; Balick \& al., 1987). As a matter of thorough presentation, give group values (means), error variance (standard deviations and sample sizes), and statistical tests (multiple comparisons) - see Tables 1 and 2. (4) Count the number of characters that are and that are not intermediate, and judge whether the coalescence of intermediate character states is too improbable to represent divergence in the same characters in the same direction. This can be considered a one-sided sign test of intermediate versus non-intermediate characters. Such a test will be significant at the 0.05 level when 5 out of 5 characters are intermediate, or 9 out of 11,10 out of 13 , or 12 out of 16 (Zar, 1984).

Table 1 presents a character count for the simulated phyletic series. Species 1 is intermediate between 2 and 3 in 8 characters and non-intermediate in 8 . There is no significant deviation $(P>0.05)$. The hypothesis of divergence is not rejected. Table 2 presents a character count for the simulated reticulate series. Species 6 is intermediate between 4 and 5 in 14 characters and non-intermediate in a single one. The deviation is very significant $(P<0.01)$. This pattern would not be expected from divergence, so the hypothesis of hybridity is accepted. In the case of high dominance (not shown), 12 characters were intermediate, 3 were not. This is significant $(P<0.05)$. Hybridity is accepted. The character count procedure successfully distinguishes hybridity from divergence. 
The null model for the test in step 4 above is divergent evolution in which two assumptions are made. (1) It is assumed the direction in which a character changes in one segment of a phylogeny has no bearing on the direction in which it changes in another segment: In Fig. 1, central spot size could have increased or decreased along either diagonal but whether it increased or decreased in the first diagonal would be assumed to have no influence on whether it increased or decreased in the second diagonal. (2) It is assumed that characters are independently free to evolve in either direction with respect to each other: In Fig. 1, knob pigmentation is assumed to have not been pleiotropically or functionally correlated with central spot size. If one does not wish to make the assumptions, intermediate and non-intermediate characters should still be tallied and reported as if the test were going to be done. This remains useful since, even when the assumptions are not precisely true, a preponderance of intermediate character states is still evidence against divergence in favor of hybridity. The assumptions merely enable one to make a quantitative significance statement, such as, intermediacy in 9 out of 11 characters has a less than $5 \%$ chance of resulting from divergent evolution.

\section{Discussion}

I have provided a statement and examples of how to and how not to infer hybridity from morphological intermediacy. Hybrid indices do not distinguish between divergence and hybridity. Principal components analysis does so in only a limited manner that is unduly susceptible to the influence of other factors such as dominance. Pictorialized scatter diagrams properly present the evidence for intuitive interpretation. The character count procedure described here successfully distinguishes hybridity from divergence. In presenting a case for hybridity, I advise including a diagram like Fig. 9 and a character count like Table 2.

In the systematics of hybrids and their parents, there are a series of increasingly difficult questions that might be addressed using a single data set but with different analyses: (1) Did a pattern of diversity arise through hybrid origin (hybridity)? (2) Has there been character recombination through backcrossing toward a parental species (introgression, narrowly defined)? (3) What are the frequencies of $F_{1}$ 's, backcrosses, etc. (the structure of a hybrid swarm)? Principal components, hybrid indices, and similar methods may be useful in analyzing introgression and swarm structure after one has already demonstrated that hybridization is involved to begin with. Unfortunately, methodological papers on this topic have not made it clear that the methods for showing introgression or swarm structure are not acceptable for showing hybridity itself, and that before even trying to show introgression or swarm structure it is necessary to first show hybridity (e.g., Anderson, 1949; Hatheway, 1962; Namkoong, 1966; Wells, 1980; Pimentel, 1981; Adams, 1982; Brockmann, 1987).

In connection with this lack of clarity in the methodological literature, empirical reports frequently use analyses erroneously, or at least seem to. There are many instances in which a naive reader would think that hybrid indices or principal components were being used to demonstrate hybridity, and there are many instances where a better approach could have been used than was used (e.g., Kirkbride, 1976; Sytsma \& Pippen, 1985; Johns, 1987; Bateman \& Farrington, 1987; Kephart \& al., 1988; Parnell \& Simpson, 1988; Ness \& al., 1990). It is often unclear whether a multivariate analysis is being used (incorrectly) to show hybridity or whether hybridity has already been established in an earlier part of a paper and the multivariate analysis is being used to 
show introgression or swarm structure - authors would do well to declare their exact intentions. Although the presentation of evidence for hybridity often seems poor, reports of hybridity are probably nevertheless correct because researchers do understand intuitively the difference between character-by-character intermediacy and overall intermediacy.

\section{Acknowledgements}

My ideas were inspired by studying Ribes hybrids with M. Mesler. C. Kurtz helped with the programing. For manuscript comments, I am also grateful to H. Wagner, R. Sokal, J. Thomson, C. Janson, and P. Teese. Support was provided by a National Science Foundation Graduate Fellowship. This is contribution 796 in Ecology and Evolution from the State University of New York at Stony Brook.

\section{Literature cited}

Adams, R. P. 1982. A comparison of multivariate methods for the detection of hybridization. Taxon 31: 646-661.

Anderson, E. 1949. Introgressive hybridization. Hafner, New York.

Anonymous, 1985. SAS User's Guide: Statistics, Version 5. SAS Inst., Inc., Cary, NC.

$\rightarrow$ Balick, M. J., Anderson, A. B. \& Tadeu de Medeiros-Costa, J. 1987. Hybridization in the babassu palm complex. II. Attalea compta $\times$ Orbignya oleifera (Palmae). Brittonia 39: 26-36.

Bateman, R. M. \& Farrington, O. S. 1987. A morphometric study of $\times$ Orchioceras bergonii (Nauteuil) Camus and its parents (Aceras anthropophorum (L.) Aiton f. and Orchis sinia Lamarck) in Kent. Watsonia 16: 397-407.

Brockmann, C. 1987. Evaluation of some methods for analysis, exemplified by hybridization in Argyranthemum (Asteraceae). Nord. J. Bot. 7: 609-630.

Dancik, B. P. \& Barnes, B. V. 1975. Multivariate analyses of hybrid populations. Naturaliste Canad. 102: 835-843.

Davis, P. H. \& Heywood, V. H. 1963. Principles of angiosperm taxonomy. Oliver \& Boyd, Edinburgh \& London.

$\rightarrow$ Funk, V. A. 1985. Phylogenetic patterns and hybridization. Ann. Missouri Bot. Gard. 72: 681-715.

$\rightarrow$ Gay, P. A. 1960. A new method for the comparison of populations that contain hybrids. New Phytol. 59: 218- 226.

$\rightarrow$ Goodman, M. M. 1967. The identification of hybrid plants in segregation populations. Evolution 21: 334-340.

$\rightarrow$ Hatheway, W. H. 1962. A weighted hybrid index. Evolution 16: 1-10.

$\rightarrow$ Johns, T. 1987. Relationships among wild, weed and cultivated potatoes in the Solanum $\times$ ajanjuiri complex. Syst. Bot. 12: 541-552.

$\rightarrow$ Kephart, S. R., Wyatt, R. \& Parrella, D. 1988. Hybridization in North American Asclepias. I. Morphological evidence. Syst. Bot. 13: 456-473.

$\rightarrow$ Kirkbride, J. H. 1976. Confirmation of hybridization between Declieuxia fruticosa and $D$. passerina (Rubiaceae). Brittonia 28: 341-347.

$\rightarrow$ McDade, L. 1990. Hybrid and phylogenetic systematics: I. Patterns of character expression in hybrids and their implications for cladistic analysis. Evolution 44: 1685-1700.

$\rightarrow$ Namkoong, G. 1966. Statistical analysis of introgression. Biometrics 22: 488-502.

$\rightarrow$ Ness, B. D., Soltis, D. E. \& Soltis, P. S. 1990. An examination of polyploidy and putative introgression in Calochortus subsection Nudi (Liliaceae). Amer. J. Bot. 77: 1519-1531.

Parnell, J. A. N. \& Simpson, D. A. 1988. Hybridization between Polygonum mite Schrank, $P$. minus Huds. and $P$. hydropiper $\mathrm{L}$. in northern Ireland with comments on their distinction. Watsonia 17: 265-272. 
Pimentel, R. A. 1979. Morphometrics. Kendall \& Hunt, Dubuque, Iowa.

- 1981. A comparative study of data and ordination techniques based on a hybrid swarm of sand verbenas (Abronia Juss.). Syst. Zool. 30: 250-267.

$\rightarrow$ Rieseberg, L. H., Soltis, D. E. \& Palmer, J. D. 1988. A molecular reexamination of introgression between Helianthus annuus and H. bolanderi (Compositae). Evolution 42: 227-238.

$\rightarrow$ Smouse, P. E. 1972. The canonical analysis of multiple species hybridization. Biometrics $28:$ 361-371.

$\rightarrow$ Sytsma, K. J. \& Pippen, R. W. 1985. Morphology and pollination biology of an intersectional hybrid of Costus (Costaceae). Syst. Bot. 10: 353-362.

$\rightarrow$ Wagner, W. H. 1969. The role and taxonomic treatment of hybrids. BioScience 19: 785-789+795.

- 1983. Reticulistics: The recognition of hybrids and their role in cladistics and classification. Pp. 63-79 in: Platnick, N. I. \& Funk, V. A (ed.), Advances in cladistics, 2. Columbia University Press, New York.

Warwich, S. I, Bain, J. F., Wheatcroft, R. \& Thompson, B. K. 1989. Hybridization and introgression in Carduus nutans and C. acanthoides reexamined. Syst. Bot. 14: 476-494.

Wells, H. 1980. A distance coefficient as a hybridization index: An example using Mimulus longiflorus and M. flemingii (Scrophulariaceae) from Santa Cruz Island, California. Taxon 29: 53-65.

Zar, J. H. 1984. Biostatistical analysis. Prentice-Hall, Englewood Cliffs. 